\title{
Generic Control Allocation Toolbox for Preliminary Vehicle Design
}

\author{
Jing Pei * \\ NASA Langley Research Center, Hampton, Virginia, 23681 \\ George Z. Bassett ${ }^{\dagger}$ \\ University of Texas at Austin, Austin, Texas, 78712 \\ James Grisham $\ddagger$ \\ University of Texas at Arlington, Arlington, Texas, 76019 \\ Peter Finch $\S$ \\ Stanford University, Stanford, CA, 94305 \\ Matthew Toniolo 9 \\ Analyical Mechanics Associates, Hampton, Virginia, 23681 \\ Luke Miller "and Bandu Pamadi ** \\ NASA Langley Research Center, Hampton, Virginia, 23681
}

This paper describes the development and application of a Generic Control Allocation Toolbox developed at NASA Langley Research Center (LGCAT) intended to aid engineers during the preliminary design phase of an aerospace vehicle. The static controllability space in the forms of a Theoretical Attainable Moment Set, $\Phi$, or Theoretical Attainable Force Set, $\phi$ is difficult to visualize for modern vehicles with multiple types of redundant control effectors. The objective of LGCAT is to provide system engineers and designers early in the vehicle design phase with quick insights on how control effector parameters such as quantity, sizing, location, orientation, redundancy, etc., affect the overall controllability and other performance metrics. Having such information in hand allows system engineers to make more informed decisions on overall mission objectives such as performance vs. reliability vs. cost, etc. early in a vehicle design phase and reduce the number of iterations necessary in the design and analysis cycles. LGCAT can accept a variety of control effector types including aerodynamic surfaces, rotors, thrust vector control (TVC) engines, and reaction control systems (RCS). LGCAT is MATLAB based, user friendly, and is capable of performing the analysis in the Graphical User Interface (GUI) or script mode. Current add-on features include interfacing with engineering level codes such as Vehicle Sketch Pad (VSP) and generating the corresponding $\Phi$ and $\phi$ for an arbitrary vehicle design. These capabilities potentially make LGCAT an integral part of the preliminary design phase for any vehicle.

\footnotetext{
*Aerospace Engineer, Vehicle Analysis Branch

${ }^{\dagger}$ Currently: Graduate Student, Georgia Institute of Technology

$¥$ PhD Student, University of Texas at Arlington

$\S$ Graduate Student, Stanford University

๑ Aerospace Engineer, Analytical Mechanics Associates

\| Pathway Student, Flight Dynamics Branch

${ }^{* *}$ Retired Aerospace Engineer, Vehicle Analysis Branch
} 


\section{Introduction}

Typical modern aerospace vehicles, ranging from aircraft to launch vehicles to spacecraft, have multiple redundant control effectors to meet performance and fault tolerance requirements. Figure 1 shows several well-known examples across the spectrum of aerospace vehicles: GL-10, SLS, MLAS, Space Shuttle, F-16, and Cassini Spacecraft. Depending on the vehicle flight regime (endo-atmosphere, exo-atmosphere, or both) and mission requirements, the typical control effector options are: aerodynamic surfaces, propellers, thrust vector control (TVC), reaction control system (RCS), reaction wheels (RW), control moment gyros (CMG), and torque rods. The vehicle controllability space can be viewed in the forms of: Theoretical Attainable Moment Set, $\Phi^{1-3}$ or Theoretical Attainable Force Set, $\phi$. As a vehicle design becomes more complicated with a mixture of effector types, such as with the GL-10, it is virtually impossible for the analyst to visualize $\Phi$ or $\phi$ without the aid of a numerical toolbox.

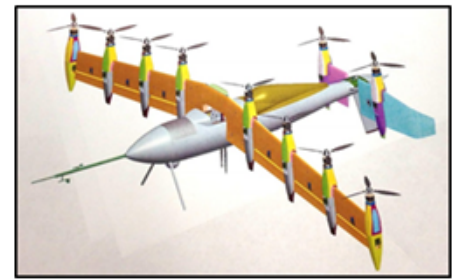

G-10 Concept

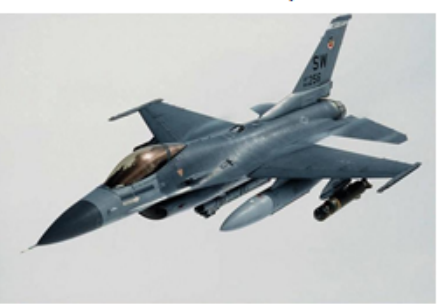

F-16 Fight Aircraft

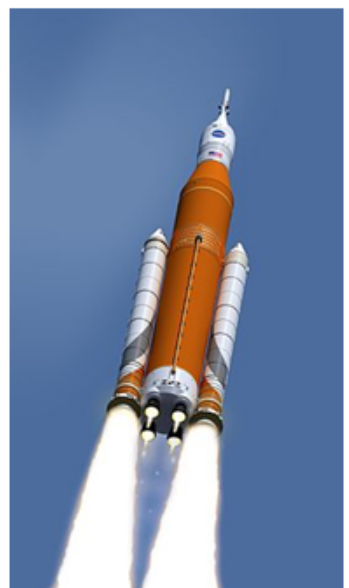

Space Launch System Rocket

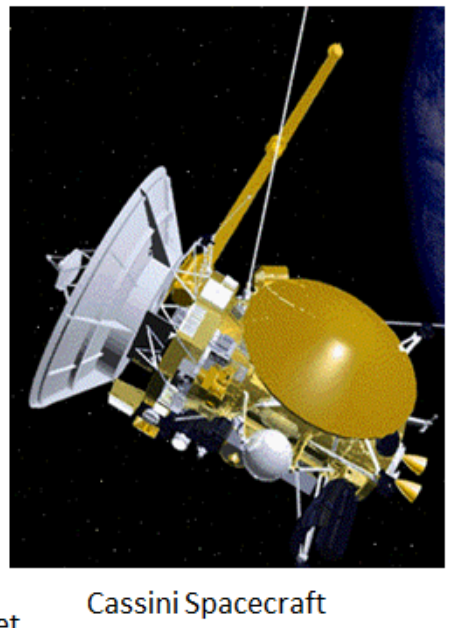

Cassini Spacecraft

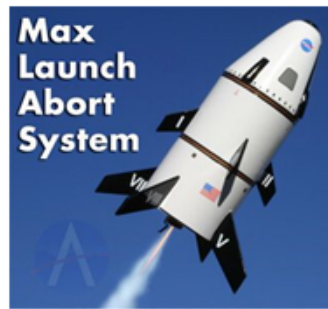

Launch Abort System

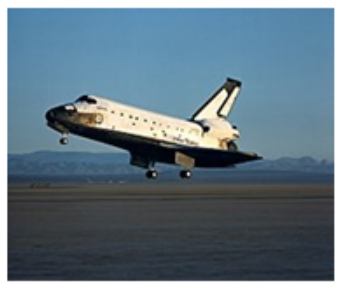

Space Shuttle

Figure 1: Examples: Aerospace Vehicles with Redundant Effectors

The control allocation problem addresses how control effectors of a vehicle should be positioned in order to achieve a certain command (usually three-axis moment or force) from the output of some control law. It is typically defined as: ${ }^{3}$ given a column matrix of desired moments, $m_{\text {des }}$, control effectiveness matrix, $B$, lower and upper limits of each effector $\left(u_{\min }\right.$ and $\left.u_{\max }\right)$, find $u$ that satisfies:

$$
\begin{gathered}
B u=m_{\text {des }} \\
\text { s.t. } \\
u_{\min } \leq u \leq u_{\max }
\end{gathered}
$$

The number of control effectors is generally greater than the dimension of the desired force or moment and it is usually up to the Guidance Navigation \& Control (GN\&C) team to develop the "best" algorithm that minimizes some objective function ${ }^{1-5}$ (e.g., error between the commanded and actual moments in addition to the total effector usage) while satisfying certain constraints (e.g., servo maximum position and rate limits or RCS thruster minimum and maximum on-time). This level of analysis is often performed much later in the vehicle design phase.

The importance of analyzing static control allocation metrics such as $\Phi$ or $\phi$, early in a vehicle design phase is often overlooked. It is crucial for the system designers to have insights into how parameters such as control effector effectiveness, quantity, location, size, and orientation influence the overall admissible control space. Having such knowledge allows the engineer to make informed decisions about crucial matters such as: 1) the minimal suite of control effectors required to still meet the desired system performance metrics, 2) the extent of sufficiency in three-axis control in the event of an effector failure, 3) ratio of control authority compared to the disturbance moment set (e.g., the Space Shuttle heritage criteria for the RCS system requires the control torque to exceed all known disturbance torque by a factor of two), 4) optimal location for a set 
of RCS thrusters given a set of constraints. Quick assessments of these types of issues early in the vehicle concept development phase can mitigate schedule delays and reduce life-cycle costs.

The motivation for the development of the NASA Langley Generic Control Allocation Toolkit (LGCAT), was to provide a general framework that incorporates key aspects of flight controls early in a vehicle design phase. The easy-to-use tool allows the analyst to perform quick trade studies and rapidly evaluate various effector configurations by comparing the corresponding $\Phi$ or $\phi$. The modularity of LGCAT allows for a mixture of control effector types and gives the analyst the options to either supply the parameters associated with individual effectors or enter a pre-computed control effectiveness matrix, $B$. Generalized inverse solutions to the control allocation problem are the most commonly used approaches due to their linearity and low computational cost. ${ }^{2,3}$ With that in mind, there is a built-in pseudo inverse option that allows the analyst to compare the particular attainable moment set, ${ }^{3,6} \Phi^{*}$ to $\Phi$. To the best of authors' knowledge, Glaze and Durham first developed a similar MATLAB-based Control Allocation Toolbox ${ }^{6}$ (CAT). CAT is GUI driven and allows the user to input a pre-computed control effectiveness matrix and compare $\Phi^{*}$ from pseudo-inverse or direct allocation schemes with $\Phi$. However, the control effectiveness matrix is not always known especially for a configuration with a mixture of control effector types, e.g. TVC with aero surfaces. LGCAT works around this issue by giving the analyst the option to enter the control effector parameters, assemble the control effectiveness matrix internally, and subsequently compute $\Phi$ or $\phi$.

There is other related control allocation open-source software such as the MATLAB-based Quadratic Programming Control Allocation Toolbox ${ }^{7}$ (QCAT). QCAT focuses on various control allocation methods including: sequential least squares, weighted least squares, minimal least squares, interior point, cascading generalized inverses, fixed-point iterations, direct allocation, and dynamic allocation. Similar to CAT, QCAT is only capable of modeling effectors which are described in terms of control effectiveness matrix. QCAT is geared towards design of a specific control allocation scheme which serves an excellent complimentary tool to LGCAT. The rest of the paper is organized as follows: Section II provides an overview of the LGCAT framework including: underlying assumptions, available control effector types, inputs, and the possible analysis outputs. Section III compares results from LGCAT to those published in open literature. Section IV highlights several examples in which LGCAT can be beneficial in vehicle preliminary design.

\section{LGCAT Description}

The overall framework of LGCAT is presented in this section. Thus far, the program allows the analyst to examine a vehicle configuration with any combination of four control effector types (classes): aero surface, reaction control system (RCS), aircraft propeller (rotor), and thrust vector control (TVC). It is assumed that each effector produces a local force, and by virtual of being positioned at some distance away from the center of mass, a moment can be produced. In certain applications, the quantities of interest could be impulse or angular impulse. Given an expression which relates an effector input $(x)$ to a force, $\mathbf{F}(x)$, moment $\mathbf{M}(x)$, impulse $\mathbf{J}(x)$, and angular impulse $\mathbf{L}(x)$ are computed as follows:

$$
\begin{aligned}
\mathbf{M}(x) & =\mathbf{r} \times \mathbf{F}(x) \\
\mathbf{J}(x) & =\int_{0}^{t_{\mathrm{f}}} \mathbf{F}(x) d t \\
\mathbf{L}(x) & =\int_{0}^{t_{\mathrm{f}}} \mathbf{M}(x) d t
\end{aligned}
$$

where $\mathbf{r}$ is a position vector which represents the location of the control effector relative to the center of mass. Evaluating these quantities over the set of all admissible control deflections, $\Omega$ and obtaining the convex hull, ${ }^{3}$ quantities such as the theoretical attainable moment set, $\Phi$, theoretical attainable force set, $\phi$, theoretical attainable angular impulse set, $\Phi^{\prime}$, and theoretical attainable impulse set, $\phi^{\prime}$ can be readily obtained. 


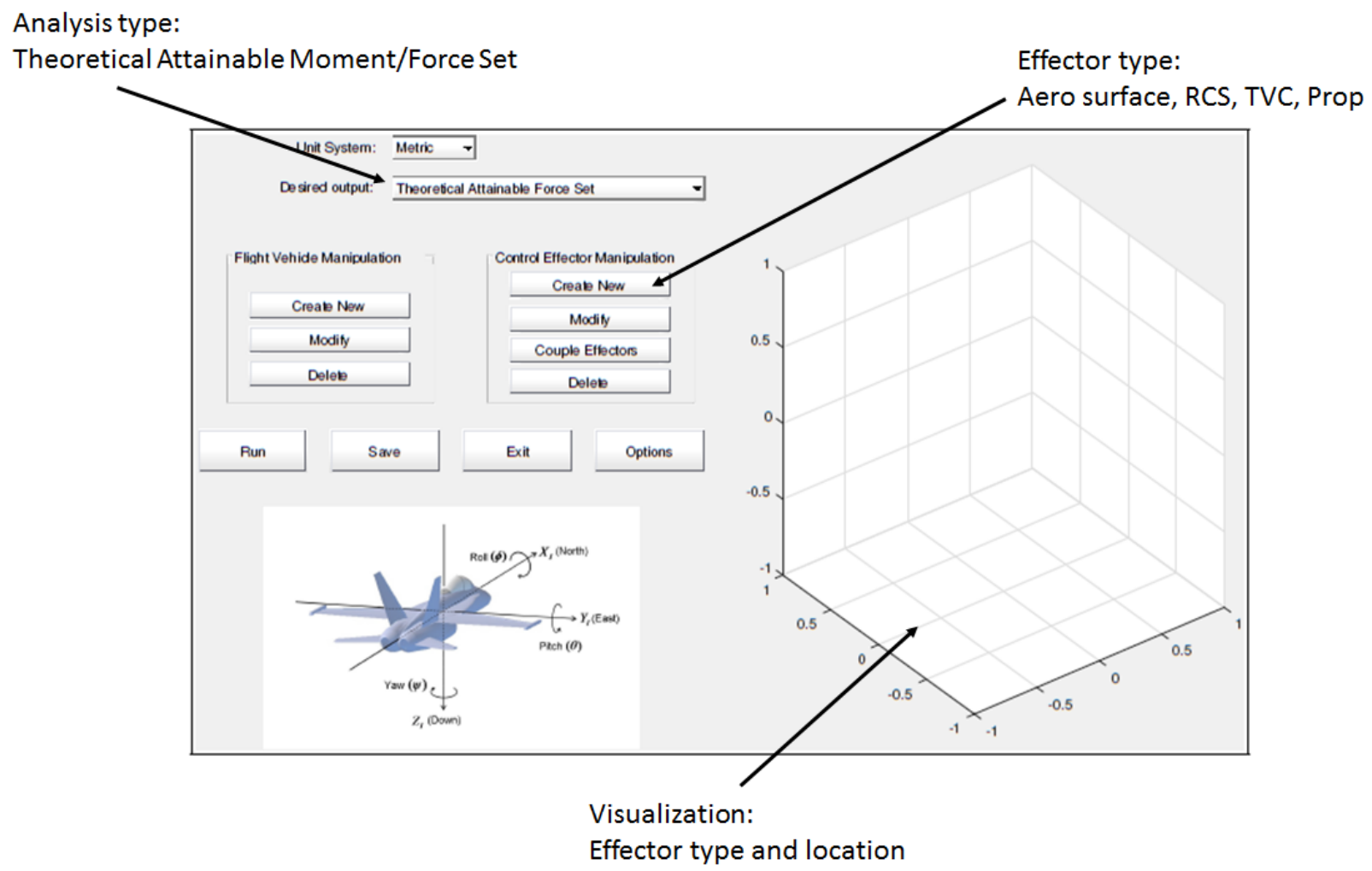

Figure 2: LGCAT GUI Main Window

Figure 2 is a snapshot of the main analysis window. The "desired output" dropdown option allows the user to select the desired analysis output: $\Phi, \phi, \Phi$ ', or $\phi$ '. The "control effector manipulation" buttons yield a popup screen that allows the user to edit the effector parameters such as displacement limits, effectiveness and locations. The visualization screen allows the user to visualize the locations and types of control effectors (color-coded) with respect to the vehicle center of mass. Once the analyst is done entering all the effectors, pressing the "run" button yields the convex hull for any one of the desired outputs. Since the pseudo inverse solution is by far the most common solution to the control allocation problem, LGCAT has a built-in option that computes $\Phi^{*}$ and $\phi^{*}$, and allows the analyst to visually compare the convex hull from the pseudo inverse solution with the theoretical maximum.

\section{A. Assumptions}

In order to develop a generic tool which is capable of handling several types of control effectors, certain simplifying assumptions were made.

- Static controllability analysis. Only the position limits $\left(u_{\min }\right.$ and $\left.u_{\max }\right)$ of the effectors are considered. LGCAT focuses primarily on obtaining the theoretical attainable moment or force sets $\Phi$ and $\phi$. How the effectors may have arrived at those positions (rate limit, etc) is not considered.

- Force for a given control input is time invariant. Using this assumption, Eq. (3) and (4) become

$$
\begin{aligned}
\mathbf{J}(x) & =\mathbf{F}(x) t_{\mathrm{f}} \\
\mathbf{L}(x) & =\mathbf{M}(x) t_{\mathrm{f}}
\end{aligned}
$$

- Integrations for impulse and angular impulse are performed for a single second. In the case of vehicles that only contain RCS thrusters, integrations for impulse and angular impulse are integrated for each RCS thruster's max on-time.

- Thrust vector control (TVC) implementation assumes that the engine has two degrees-of-freedom (DOF) and can only pivot in the $y-z$ plane. 


\section{B. Effector Types}

A brief description of the the four types of effectors implemenented thus far in LGCAT is provided in this section: aero surface, reaction control system, aircraft propeller (rotor), and thrust vector control.

\section{Aero surface}

Pure aerodynamic control effectors generally deflect some flap about a hinge line that generates a local aerodynamic force by modifying the flow over the surface. By virtue of being positioned at some distance from the center of mass, an aerodynamic moment can be generated. For moments (roll, pitch, yaw), the control effectiveness matrix for $m$ aero surfaces may be represented as

$$
\mathbf{B}=\left[\begin{array}{llll}
\frac{\partial M_{1}}{\partial u_{1}} & \frac{\partial M_{1}}{\partial u_{2}} & \ldots & \frac{\partial M_{1}}{\partial u_{m}} \\
\frac{\partial M_{2}}{\partial u_{1}} & \frac{\partial M_{2}}{\partial u_{2}} & \cdots & \frac{\partial M_{2}}{\partial u_{m}} \\
\frac{\partial M_{3}}{\partial u_{1}} & \frac{\partial M_{3}}{\partial u_{2}} & \cdots & \frac{\partial M_{3}}{\partial u_{m}}
\end{array}\right]
$$

Given a column matrix of surface deflections, the net moment on the flight vehicle would be computed as follows:

$$
\mathbf{M}=\mathbf{B} \mathbf{u}
$$

where $\mathbf{u}$ is a column matrix of inputs (deflections for aero surfaces) which can be written as $\left\{u_{1}, u_{2}, \ldots, u_{m}\right\}^{\top}$. Given this approach to modeling aero surfaces, a single aero surface can be represented by the corresponding column in the control effectiveness matrix along with limits on the possible deflections. To obtain $\Phi$, LGCAT gives the analyst the option to either input the control derivatives in the form of a control effectiveness matrix, $\mathbf{B}$, or specify the partials of force vs. deflection along with the position vector from the control surface to the center of mass.

\section{Reaction control system}

Reaction control systems (RCS) are modeled using a force vector and thruster on-time. The input to the RCS module is binary, either 0 for off or 1 for on. That is, the thruster either produces zero force or it produces a force equivalent to $\mathbf{f}$ if it is on. Time becomes important when computing impulse and angular impluse, which represent integrals of force over time and moment over time, respectively. Currently, these integrals are obtained over one second for the sake of simplicity. In cases where the RCS thrusters on-time limit is less than one second, the following logic is used to compute $\mathbf{J}$ :

if $t_{\mathrm{on}}<t_{\mathrm{f}}$

$$
\mathbf{J}(x)=\mathbf{F}(x) t_{\text {on }}
$$

otherwise $t_{\mathrm{on}} \geq t_{\mathrm{f}}$ :

$$
\mathbf{J}(x)=\mathbf{F}(x) t_{\mathrm{f}}
$$

where $t_{\mathrm{f}}$ is set to one second.

\section{Aircraft Propeller}

The rotor parameters that are used to calculated force and moment are described below.

- $\hat{\mathbf{T}}$ - unit thrust vector.

- $\mathbf{r}$ - rotor position vector.

- $C_{T}$ - coefficient of thrust.

- $C_{Q}$ - coefficient of torque.

- $\Omega$ - motor angular velocity (radians per second). 
Given these parameters, the force and moment generated can be calculated using Eq. (11) and (12), respectively. For $m$ rotors, the input would be a column matrix of motor angular velocity commands, $\Omega_{m \times 1}$

$$
\begin{aligned}
\mathbf{F} & =C_{T} \Omega^{2} \hat{\mathbf{T}} \\
\mathbf{M} & =\left[C_{T}(\mathbf{r} \times \hat{\mathbf{T}})+C_{Q} \hat{\mathbf{T}}\right] \Omega^{2}
\end{aligned}
$$

\section{4. $\quad$ Thrust vector control}

Generic thrust vector control (TVC) parameters used to compute forces and moments are described below.

- $\psi$, engine deflection in pitch direction ( $\mathrm{rad}$ )

- $\phi$, engine deflection in yaw direction (rad)

- $\mathbf{F}_{\mathbf{o}}$, force vector for un-deflected engine

- $\mathbf{r}$, position vector from the $\mathrm{CM}$ to engine

Generally, the engine has some undeflected force vector, $\mathbf{F}_{\mathbf{o}}$. It is located at some position vector away from the CM, r, and has two degrees-of-freedom (DOF) which allowes to pivot (gimbal) about the y and $\mathrm{z}$ axes of the vehicle. The engine is usually driven by a pair of linear actuators and its range of travel is limited to be within a constraint boundary prescribed by an ellipse normal to the undeflected thrust direction. The engine deflection limits in the pitch and yaw directions, $\psi$ and $\phi$ describes the shape of the ellipse. Two sequential rotations about the $\mathrm{z}$ and $\mathrm{y}$ axes can be made to rotate the undeflected force vector to some desired direction subjected to the elliptical constraint shown in Eq. 13 and 14. For a given pitch and yaw command, if the value of Eq. 14 is greater than unity then the constraint has been violated. Hence, the command is considered inadmissible and the force does not contribute to the attainable set. If the value is less than or equal to 1 then the command is considered admissible and the resultant force vector contributes to the attainable set. These sets of conditionals can be seen in Eq. 15.

$$
\begin{aligned}
& \mathbf{F}_{\text {check }}=\left[\begin{array}{ccc}
\cos (\phi) & 0 & \sin (\phi) \\
0 & 1 & 0 \\
-\sin (\phi) & 0 & \cos (\phi)
\end{array}\right]\left[\begin{array}{ccc}
\cos (\psi) & -\sin (\psi) & 0 \\
\sin (\psi) & \cos (\psi) & 0 \\
0 & 0 & 1
\end{array}\right] \mathbf{F}_{\mathbf{o}} \\
& \text { ellipse }=\frac{F_{\text {check,y }}^{2}}{\psi_{\text {lim }}^{2}}+\frac{F_{\text {check }, z}^{2}}{\phi_{\text {lim }}^{2}}
\end{aligned}
$$

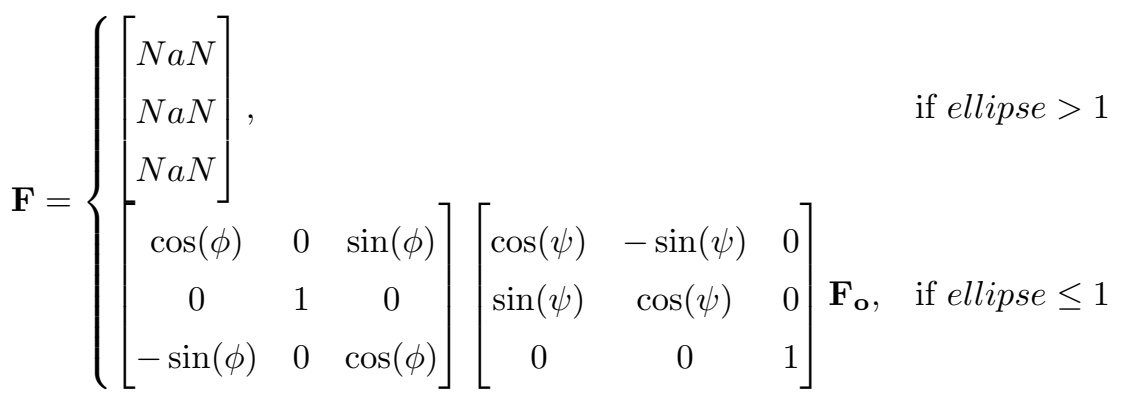

The resultant moment can be readily computed in Equation 16.

$$
\mathbf{M}=\mathbf{r} \times \mathbf{F}
$$

\section{Pseudo-Inverse Control Allocation Method}

The Moore-Penrose Pseudo-Inverse is a popular solution ${ }^{3}$ to the control allocation problem. It is generally preferred due to its linearity and speed for real-time implementation. The pseudo-inverse allocation method minimizes the sum of the squares of the control commands $\left(\mathbf{u}^{T} \mathbf{u}\right)$ subject to the constraint that $B \mathbf{u}=\mathbf{a}_{\mathbf{d}}$ where $B$ is the control effectiveness matrix, $\mathbf{a}_{\mathbf{d}}$ is the given control command, and $\mathbf{u}$ is the control commands. ${ }^{3}$ 
This is shown in Equation 17. The pseudo-inverse allocation algorithm however does not account for limits on the control effectors.

$$
\mathbf{u}=B^{T}\left[B B^{T}\right]^{-1} \mathbf{a}_{\mathbf{d}}
$$

Due to the different mechanisms for which each type of effector produces a moment or force, the construction of a general B-matrix capable of handling various combinations of effectors proved to be most challenging. With the latest version of the software, it is capable of obtaining the pseudo inverse solution to $\Phi$ and $\phi$ for any combinations of aero surface, rotors, and TVC. The RCS class stands by itself in obtaining the pseudo inverse solution for $\Phi$ ' and $\phi$ ' because of its discrete nature.

\section{Validation Cases}

A series of "check cases" was performed that compared the outputs of LGCAT with results published in the literature. Comparisons are qualitative in terms of the shape of the attainable sets and quantitative in terms of volumes of the attainable sets.

\section{A. Aero surface}

The aero surface control effector was validated by reproducing the results from Glaze and Durham. ${ }^{6}$ This example is based on a linear approximation of F-18 HARV aerodynamic data which was linearized about a flight condition of $10,000 \mathrm{ft}$, Mach 0.3 , at a $12.5^{\circ}$ angle-of-attack. The resulting control effectiveness matrix in terms of normalized rolling, pitching, and yawing moments can be seen below:

$$
\mathbf{B}^{\top}=\left[\begin{array}{ccc}
-4.382 \times 10^{-2} & -0.5330 & 1.100 \times 10^{-2} \\
4.382 \times 10^{-2} & -0.5330 & -1.100 \times 10^{-2} \\
-5.841 \times 10^{-2} & -6.486 \times 10^{-2} & 3.911 \times 10^{-3} \\
5.841 \times 10^{-2} & -6.486 \times 10^{-2} & -3.911 \times 10^{-3} \\
1.674 \times 10^{-2} & 0.000 & -7.428 \times 10^{-2} \\
-6.280 \times 10^{-2} & 6.234 \times 10^{-2} & 0.000 \\
6.280 \times 10^{-2} & 6.234 \times 10^{-2} & 0.000 \\
2.920 \times 10^{-2} & 1.00 \times 10^{-5} & 3.00 \times 10^{-4} \\
1.000 \times 10^{-5} & 0.3553 & 1.000 \times 10^{-5} \\
1.000 \times 10^{-2} & 1.000 \times 10^{-5} & 0.1485
\end{array}\right]
$$

The ten independent control effectors and their input limits are shown in Table 1. Using the above control effectiveness matrix and input limits for each effector, the theoretical attainable moment set $(\Phi)$ was computed using LGCAT and compared with Ref. 6. Figure 3 shows $\Phi$ obtained by LGCAT. The differences in $\Phi$ and $\Phi^{*}$ between Ref. 6 and LGCAT are within $1 \%$. 


\begin{tabular}{||clcc||}
\hline Control & Control effector & Minimum input (rad) & Maximum input (rad) \\
\hline \hline$u_{1}$ & right horizontal tail & -0.4189 & 0.1833 \\
\hline$u_{2}$ & left horizontal tail & -0.4189 & 0.1833 \\
\hline$u_{3}$ & right aileron & -0.5236 & 0.5236 \\
\hline$u_{4}$ & left aileron & -0.5236 & 0.5236 \\
\hline$u_{5}$ & combined rudders & -0.5236 & 0.5236 \\
\hline$u_{6}$ & right trailing-edge flap & -0.1396 & 0.7854 \\
\hline$u_{7}$ & left trailing-edge flap & -0.1396 & 0.7854 \\
\hline$u_{8}$ & roll thrust vectoring control & -0.5236 & 0.5236 \\
\hline$u_{9}$ & pitch thrust vectoring control & -0.5236 & 0.5236 \\
\hline$u_{10}$ & yaw thrust vectoring control & -0.5236 & 0.5236 \\
\hline
\end{tabular}

Table 1: F-18 Example, ${ }^{6}$ control effector position limits
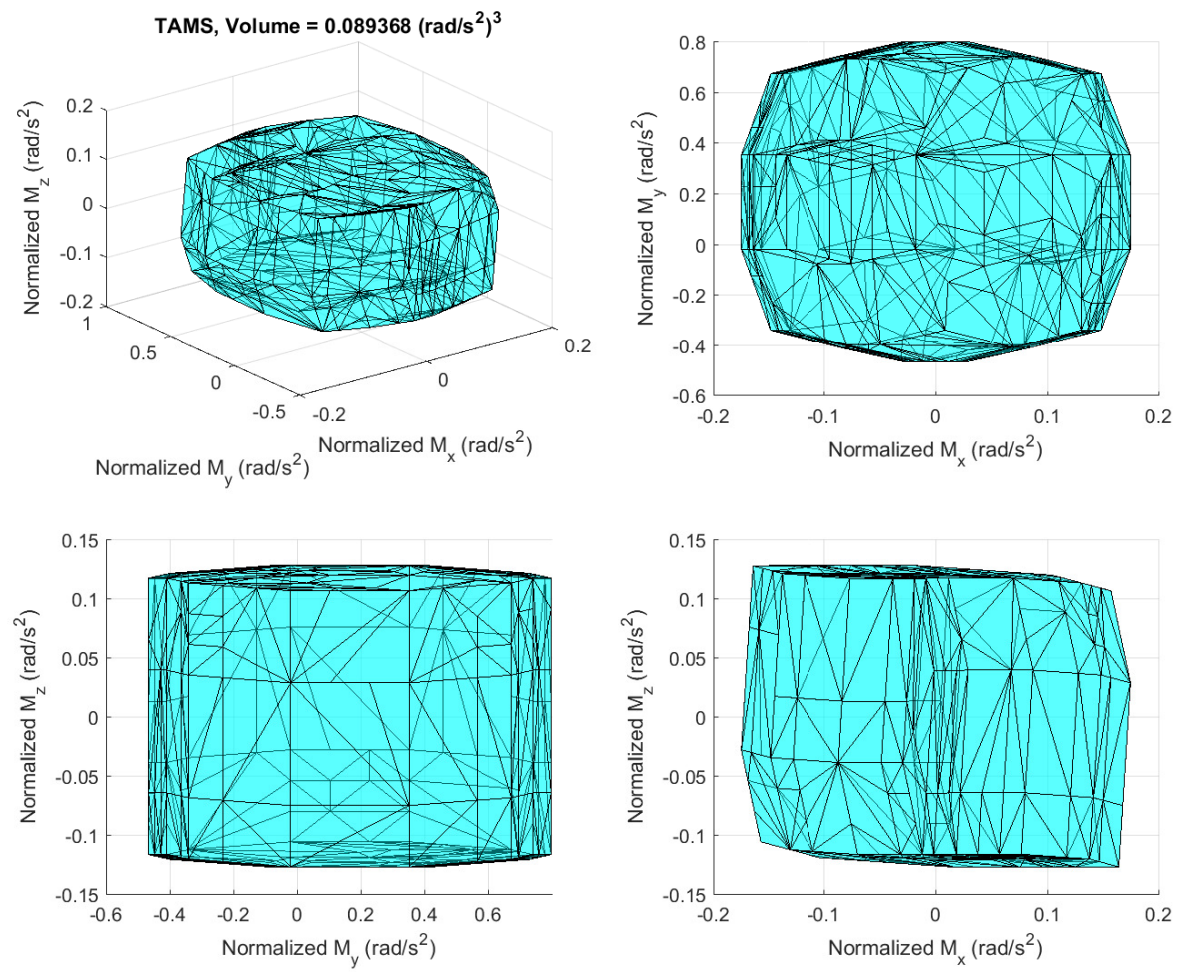

Figure 3: F-18 Example ${ }^{6}$ LGCAT, $\Phi=0.0894\left(\mathrm{rad} / \mathrm{s}^{2}\right)^{3}$

\section{B. Thrust vector control}

Thrust vector control effector results were validated using the example from Ref. 2. The example represents a reusable booster concept. The vehicle specifications and thrust vector control parameters are provided in Tables 2 and 3. Figure 4 shows $\Phi$ obtained by LGCAT. The percent differences in $\Phi$ and $\Phi^{*}$ between Ref. 2 and LGCAT are within $1.5 \%$. 


\begin{tabular}{||lc||}
\hline Parameter & Value \\
\hline \hline Mass & $44650 \mathrm{lbm}$ \\
\hline Roll Moment of Inertia, $I_{x x}$ & $0.2158 e 5 \times 10^{5} \mathrm{slug}-\mathrm{ft}^{2}$ \\
\hline Pitch Moment of Inertia, $I_{y y}$ & $3.3031 e 5 \times 10^{5} \mathrm{slug}-\mathrm{ft}^{2}$ \\
\hline Yaw Moment of Inertia, $I_{z z}$ & $3.4301 e 5 \times 10^{5} \mathrm{slug}-\mathrm{ft}^{2}$ \\
\hline Center of Mass Location (from nose) & {$\left[\begin{array}{lll}-30.6500 & 0 & -2.6400\end{array}\right]^{T} \mathrm{ft}$} \\
\hline
\end{tabular}

Table 2: Flight vehicle parameters ${ }^{2}$

\begin{tabular}{||lc||}
\hline \multicolumn{1}{|l||}{ Parameter } & Value \\
\hline \hline Thrust Plane Angle & $-3.39^{\circ}$ \\
\hline Engine Gimbal Limit & $6^{\circ}$, circular \\
\hline Engine 1 - 3 Thrust & $45000 \mathrm{lbf}$ \\
\hline Engine 1 - 3 Thrust Vector & {$\left[\begin{array}{lll}1 & 0 & 0\end{array}\right]^{T}$} \\
\hline Engine 1 Gimbal from Center of Mass & {$\left[\begin{array}{ccc}-21.21 & 0 & -2.93\end{array}\right]^{T} \mathrm{ft}$} \\
\hline Engine 2 Gimbal from Center of Mass & {$\left[\begin{array}{ccc}-21.35 & 1.45 & -0.43\end{array}\right]^{T} \mathrm{ft}$} \\
\hline Engine 3 Gimbal from Center of Mass & {$\left[\begin{array}{ccc}-21.35 & -1.45 & -0.43\end{array}\right]^{T} \mathrm{ft}$}
\end{tabular}

Table 3: Thrust vector control parameters ${ }^{2}$
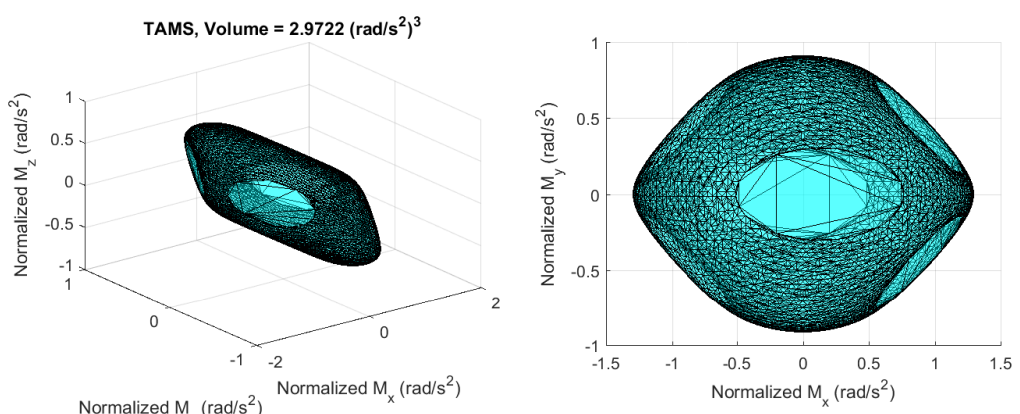

Normalized $M_{y}\left(r a d / s^{2}\right)$

Normalized $M_{x}\left(\mathrm{rad} / \mathrm{s}^{2}\right)$
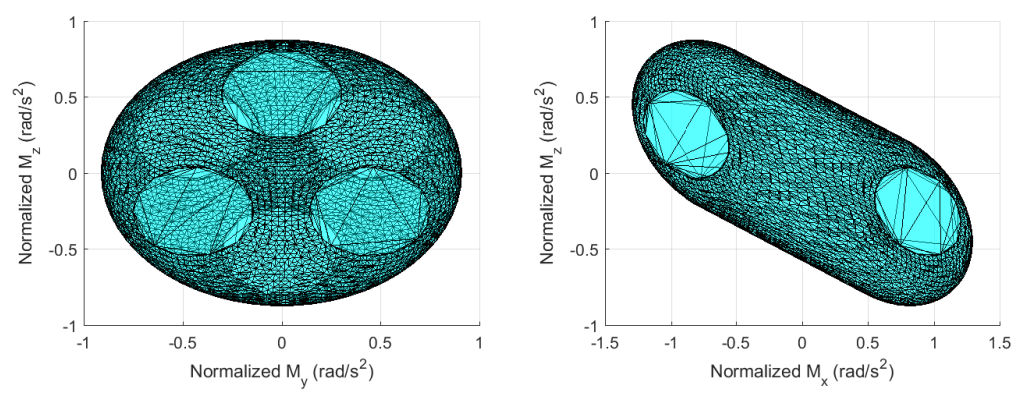

Figure 4: Reusable Booster Example ${ }^{2}$ LGCAT, $\Phi=2.9722\left(\mathrm{rad} / \mathrm{s}^{2}\right)^{3}$ 


\section{Reaction control system}

Reaction control system (RCS) results from LGCAT were validated using a 3U CubeSat configuration presented in Ref. 8. The nominal RCS configuration is designed with the thrust vectors passing through the CubeSat CM (centroid of the middle U), in which case the system provides pure translational motion of the CubeSat without affecting rotational motion. It was assumed that the thrusters have a maximum on-time of 0.2 seconds with a nominal force of $0.3 \mathrm{~N}$. Figure 5 shows the $3 \mathrm{U}$ CubeSat configuration.

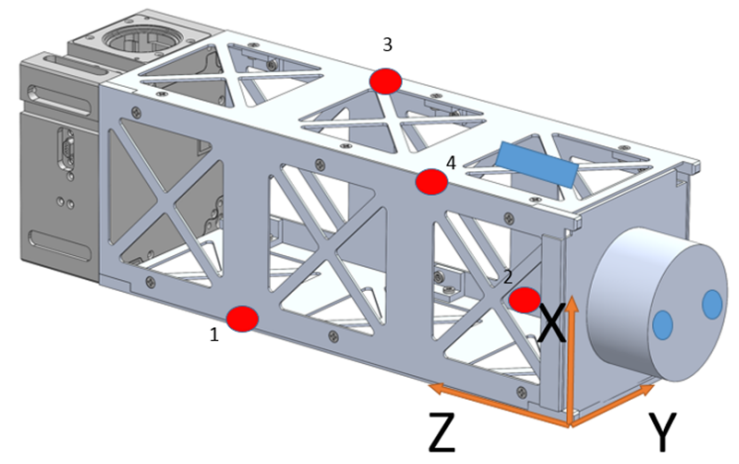

Figure 5: CubeSat Example RCS Layout ${ }^{8}$

Figure 6 shows the resultant theoretical attainable impulse set, $\phi$, of the RCS configuration provided in Ref. 8. Figure 7 shows $\phi$ computed by LGCAT. The difference between the results in the published paper with those obtain with LGCAT is less than $0.01 \%$.

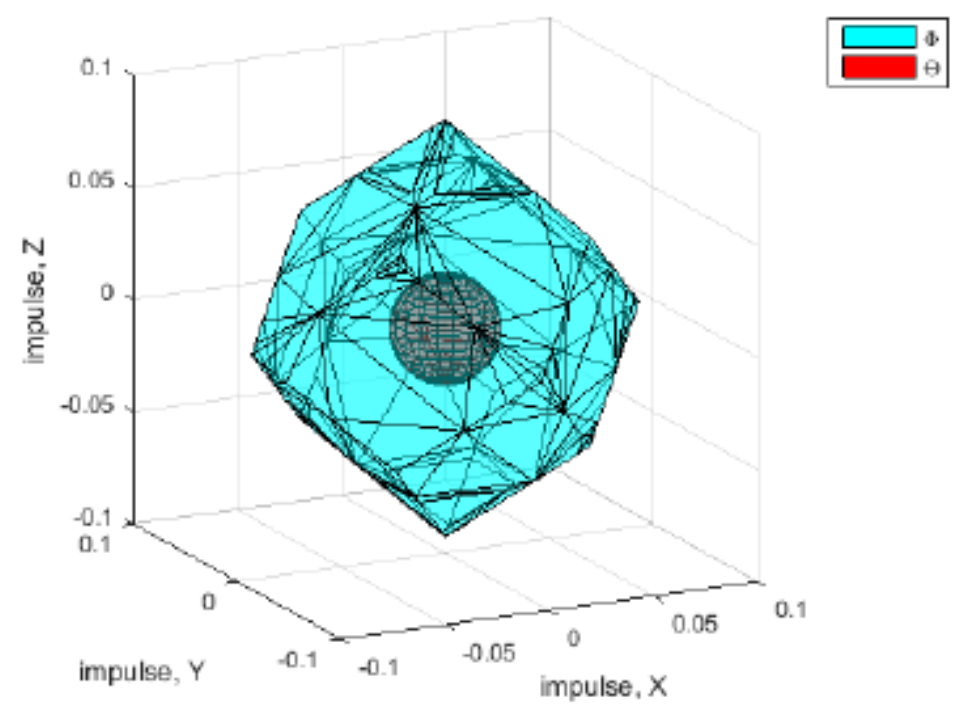

Figure 6: CubeSat RCS Example, ${ }^{8} \phi=0.0016 \mathrm{Ns}$ 

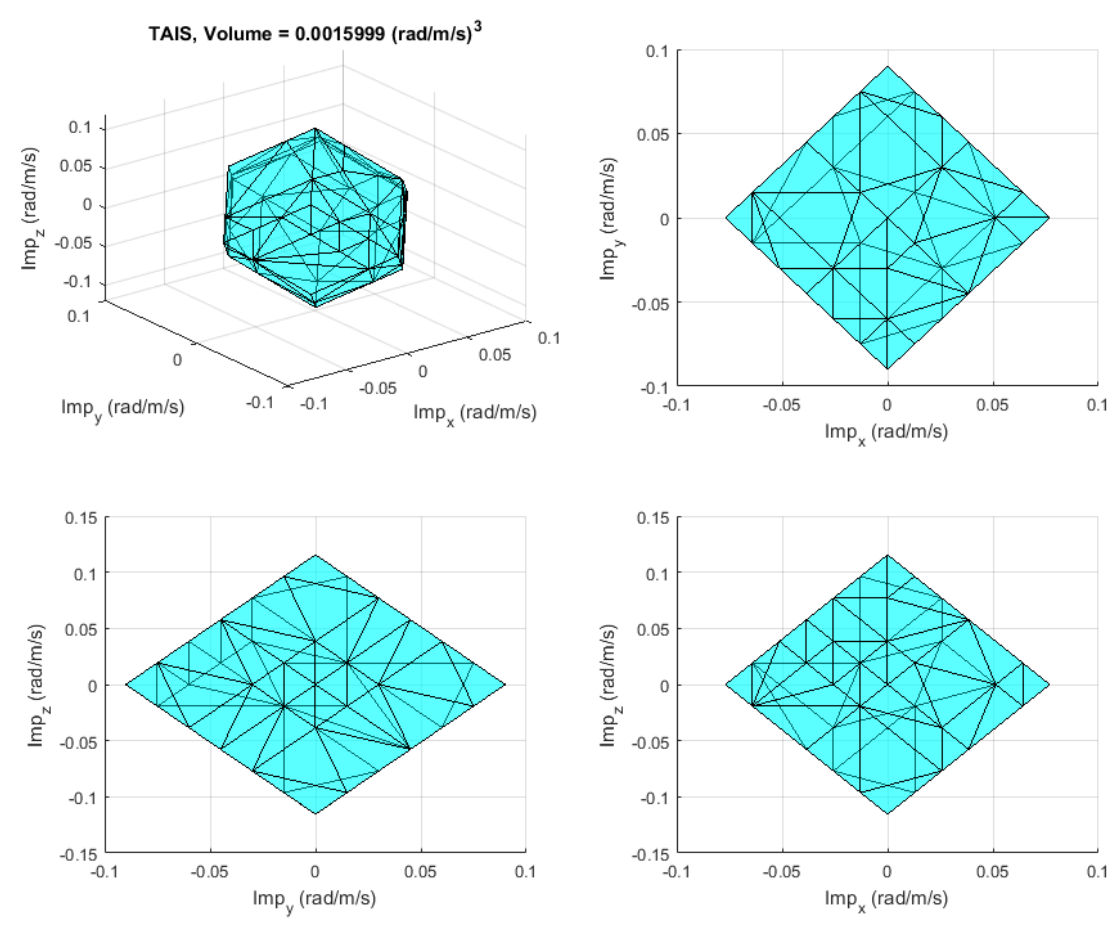

Figure 7: CubeSat RCS Example ${ }^{8}$ LGCAT, $\phi=0.00158$ Ns

\section{Application of LGCAT}

The primary function of LGCAT is to provide designers and analysts with a numerical toolbox that allows easy visualization of the static controllability space in the forms of $\Phi$ or $\phi$ for complicated vehicle configurations with a mixture of control effector types. Having such information would allow the engineer to make informed decisions to crucial design questions such as: 1) the minimal suite of control effectors necessary while stil meeting the desired performance requirements, 2) sufficient control authority in the event of a failed effector. This section highlights several applications of LGCAT in the vehicle design phase including: 1) visualizing $\Phi$ for complicated vehicle configurations such the GL-10 and space shuttle, 2) serving as a complimentary tool to engineering level codes such as Vehicle Sketch Pad (VSP), ${ }^{9}$ Athena Vortex Lattice Method (AVL), ${ }^{10}$ and missile DATCOM, ${ }^{11} 3$ ) serving as an integral part of a framework for optimizing effector design from a vehicle flight controls perspective.

\section{A. Using LGCAT to Visualize Complex Vehicle Configurations}

1. $G L-10$

GL-10 was designed at NASA Langley Research Center as an efficient hybrid-electric Vertical Take-Off and Landing (VTOL) aircraft. ${ }^{12}$ GL-10 is an extremely complicated configuration with multiple propellers and control surfaces; in addition, the entire wing and tail can both be rotated. Hence, it is virtually impossible to visualize the control moment space without the aid of a numerical tool. Figure 8 shows the three flight modes of the GL-10 design. ${ }^{12}$ 


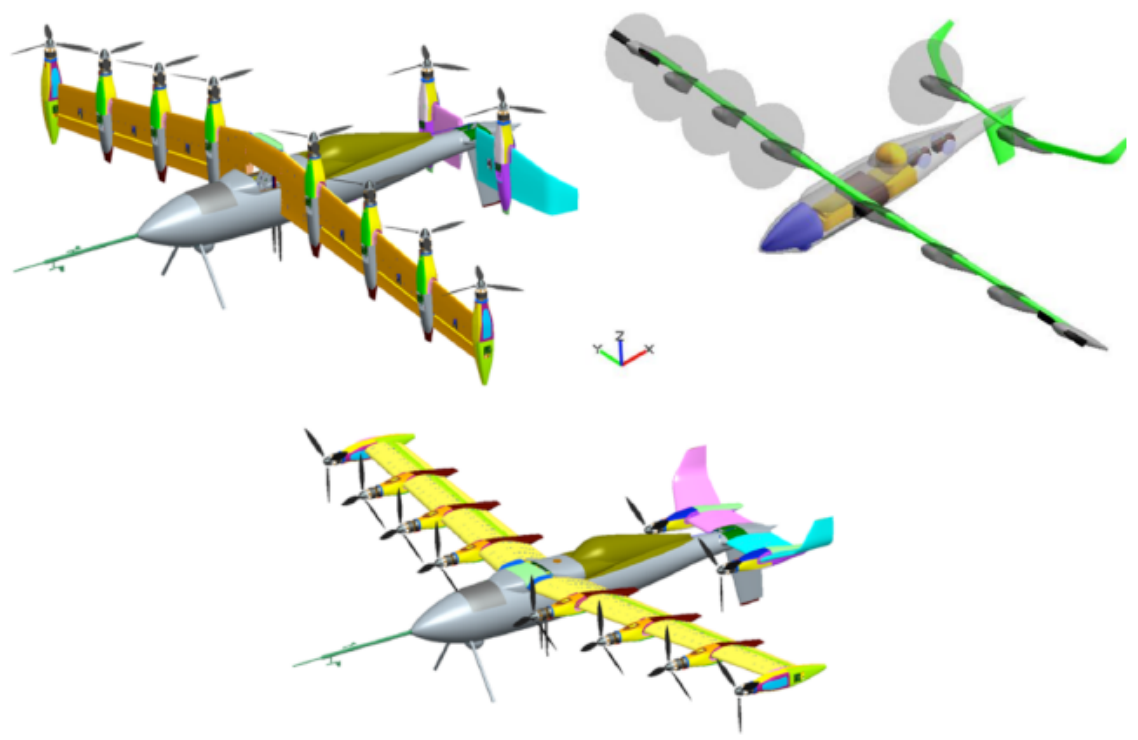

Figure 8: GL-10 modes of operation: ${ }^{12}$ hover, transition, and cruise

The moment space for the hover mode is examined here. For hover, GL-10 uses all 8 rotors on the wing and the 2 rotors located on the tail to produce thrust in addition to the three moments. The vehicle and effector parameters including the admissible limits were entered into LGCAT and the tool output the theoretical attainable moment set, $\Phi$. Under nominal operations, the configuration has ample amount of control in all three axes shown by the blue volume in Fig. 9. The superimposed red volume represents the reduced moment space, $\Phi^{\dagger}$ (subset of $\Phi$ ), in the event the far left rotor on the port side of the wing has failed. It is apparent that with the far left rotor out, the vehicle loses a significant amount of roll control. Furthermore, the shape of the moment space is no longer symmetric as expected.
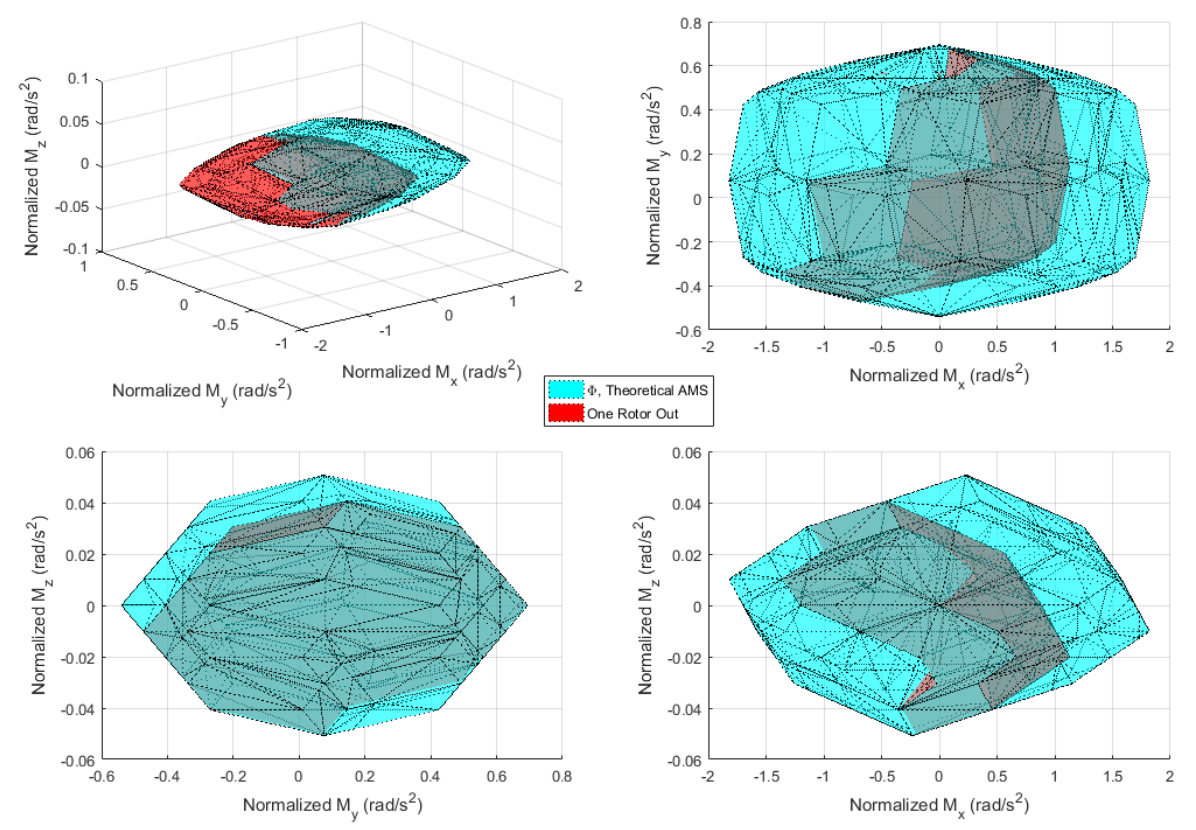

Figure 9: GL-10 Hover Mode, $\Phi$ nominal vs. and $\Phi^{\dagger}$ one rotor out 


\section{Space Shuttle's Reaction Control System}

The Space Shuttle's Reaction Control System ${ }^{13}$ consisted of 38 primary RCS thrusters and 6 Vernier RCS thrusters that were used for attitude control during flight operations such as payload insertions and docking with the International Space Station (ISS). The layout of the 44 RCS thrusters is shown in Figure 10. The RCS thrusters are split into two major sections: the forward and aft thrusters. The forward thrusters are used for on-orbit maneuvering while the aft thrusters are used for re-entry (along with the aero-surfaces).

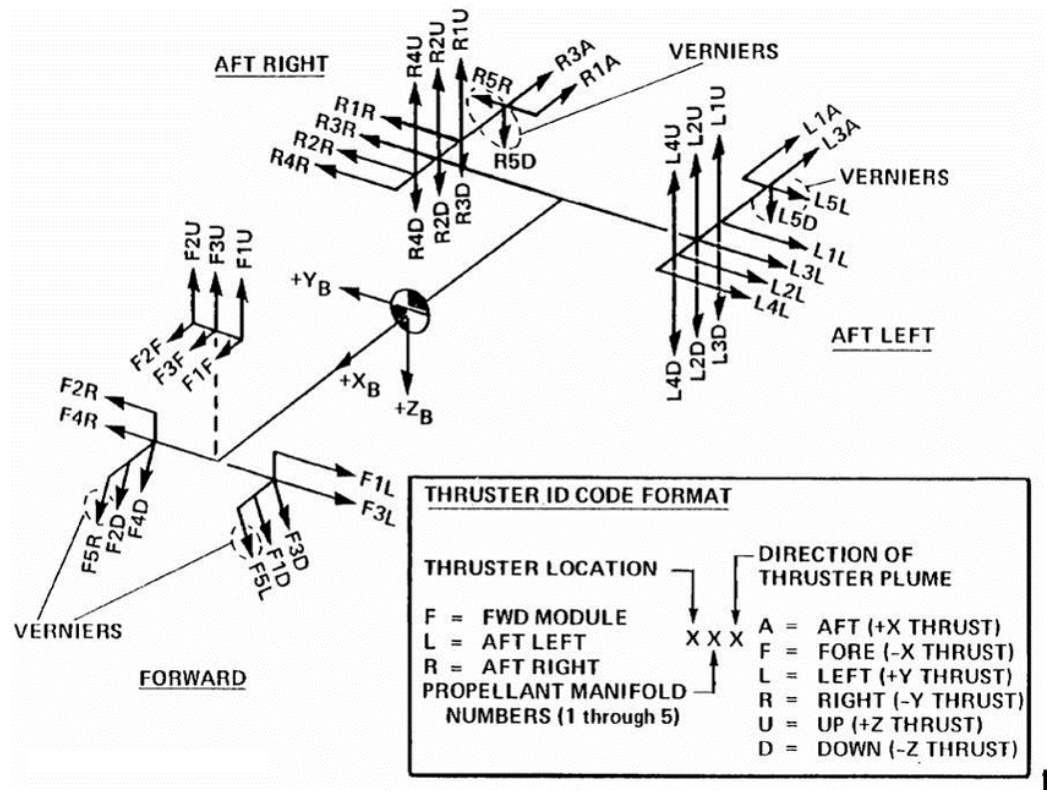

Figure 10: Layout of Space Shuttle's Reaction Control System ${ }^{13}$

Figure 11 shows the attainable moment set, $\Phi$, for Space Shuttle's RCS configuration. LGCAT allows the designer to quickly address important design questions such as the ratio of the control torque to disturbance torques. One of the GN\&C requirements was that the control torque must be at least a factor of two greater than all known disturbance torques (gravity gradient, solar radiation, atmospheric drag, etc.). With LGCAT, the analyst can co-plot the control moment set with the disturbance torque set and quickly address such requirements for such a complicated RCS configuration. 


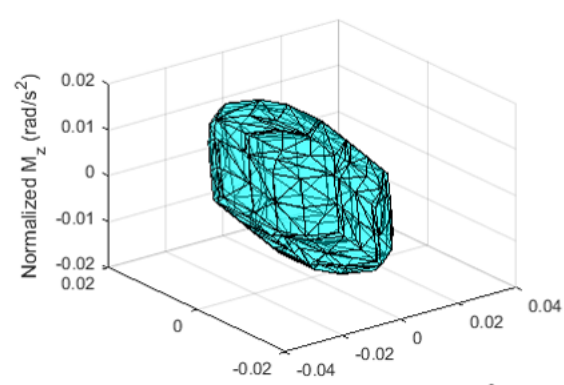

Normalized $\mathrm{M}_{\mathrm{y}}\left(\mathrm{rad} / \mathrm{s}^{2}\right)$

Normalized $\mathrm{M}_{\mathrm{x}}\left(\mathrm{rad} / \mathrm{s}^{2}\right)$

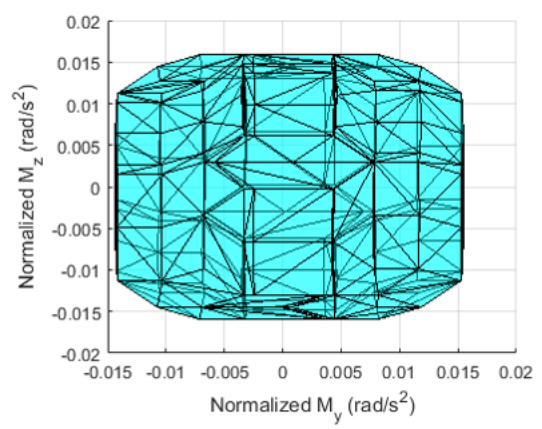

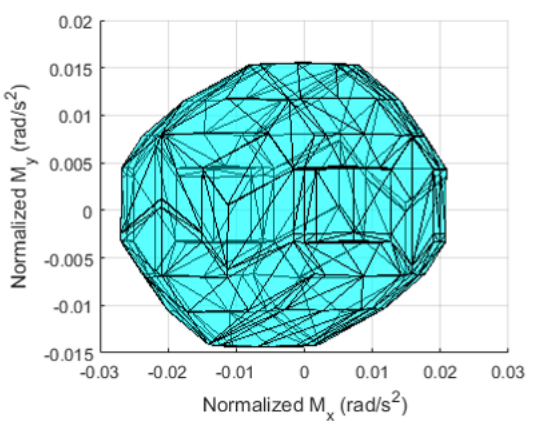

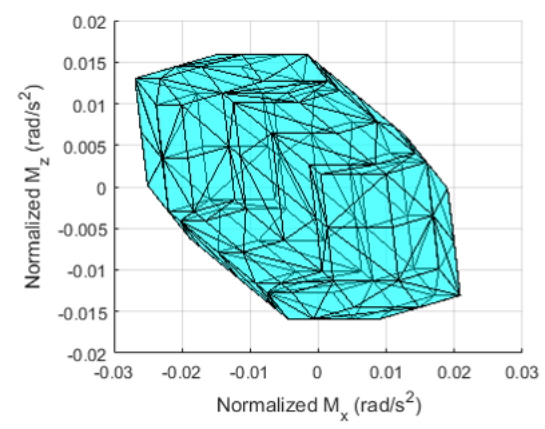

Figure 11: $\Phi$ for Space Shuttle's Reaction Control System

\section{B. Using LGCAT with Vehicle Sketch Pad}

Vehicle Sketch Pad (VSP) is a popular parametric aircraft design tool. ${ }^{9}$ VSP allows the user to create rapidly a 3D model of an aircraft and contains built-in tools to conduct preliminary sizing, aerodynamic, and mass property analysis. VSPAero is a vortex lattice solver and together with the VSP mass properties tool gives the analyst a way of rapidly generating stability and control derivatives. Coupling VSP with LGCAT can allow aircraft designers to perform quick evaluation of the vehicle controllability metrics during explorations of the configuration design space early in a design cycle. The process of creating an aircraft configuration and generating the stability derivatives in VSP, and then passing the results to LGCAT is demonstrated with an RV-7 configuration. The RV-7 has 4 control surfaces consisting of ailerons, rudder, elevator, and flaps. The control surface deflection limits are shown in Table 4. Figure 12 is a sketch of the RV-7 configuration generated in VSP.

\begin{tabular}{||clcc||}
\hline Control & Control effector & Minimum input (rad) & Maximum input (rad) \\
\hline \hline$u_{1}$ & aileron & -0.349 & 0.349 \\
\hline$u_{2}$ & rudder & -0.349 & 0.349 \\
\hline$u_{3}$ & elevator & -0.349 & 0.349 \\
\hline$u_{4}$ & flaps & 0.000 & 0.349 \\
\hline
\end{tabular}

Table 4: RV-7 Control effector position limits 


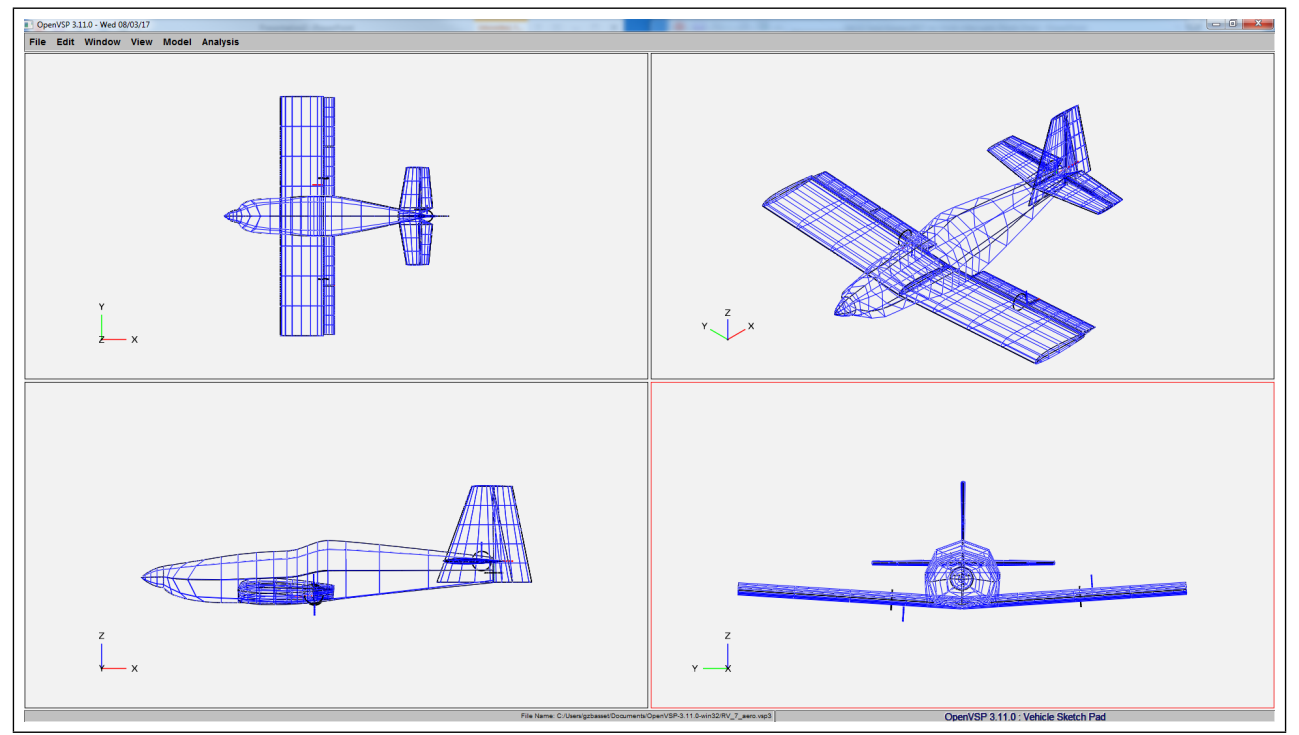

Figure 12: RV-7 in VSP

Figure 13 is an overlay of $\Phi$ at two flight conditions, $\alpha=0^{\circ}$ (blue) and $2^{\circ}$ (red). This is an excellent example of how LGCAT can be used in conjunction with engineering level tools such as VSP, ${ }^{9}$ AVL, ${ }^{10}$ and DATCOM ${ }^{11}$ in quickly assessing the vehicle controllability space at various operating conditions. This information in turn can affect design decisions such as operating flight envelope for aircraftsand UAVs or requirements for system performance in the event of an effector failure.
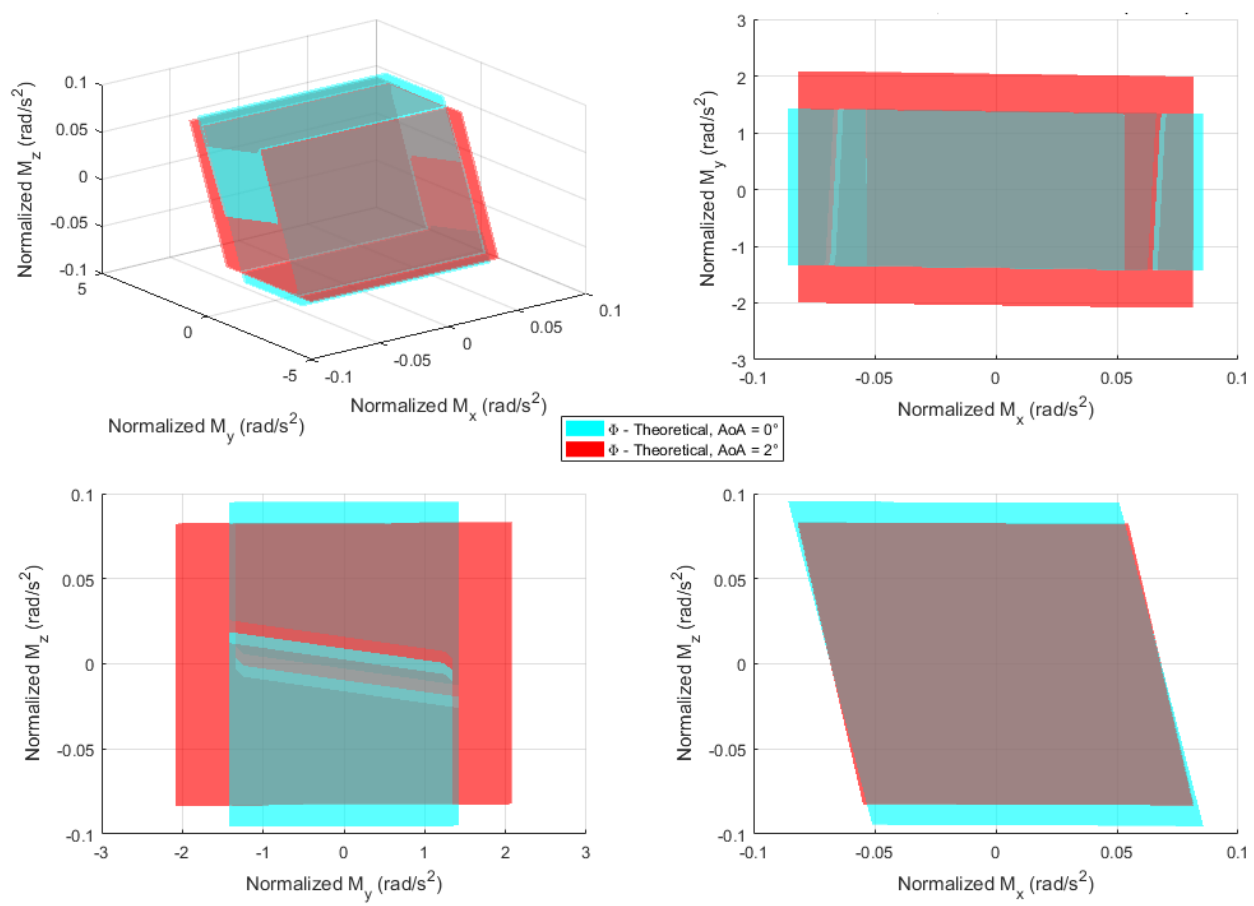

Figure 13: $\Phi$ RV-7 at $\mathrm{AoA}=0^{\circ}$ and $2^{\circ}$ 


\section{Using LGCAT to Optimize Effector Locations and Orientations}

This section describes how LGCAT can be used in a framework in which the designer can optimize the locations and orientations of control effectors in a vehicle design to maximize some objective performance parameter (i.e. $\Phi, \phi$ ) while meeting certain constraints. Figure 14 shows the schematic of such a framework. The general idea is to cast the effector design process into an optimization problem that can be readily solved by software such as MATLAB's fmincon function. ${ }^{14}$ This would ensure that an "optimal" solution has been reached, whereas conventional design approaches are usually "ad-hoc".

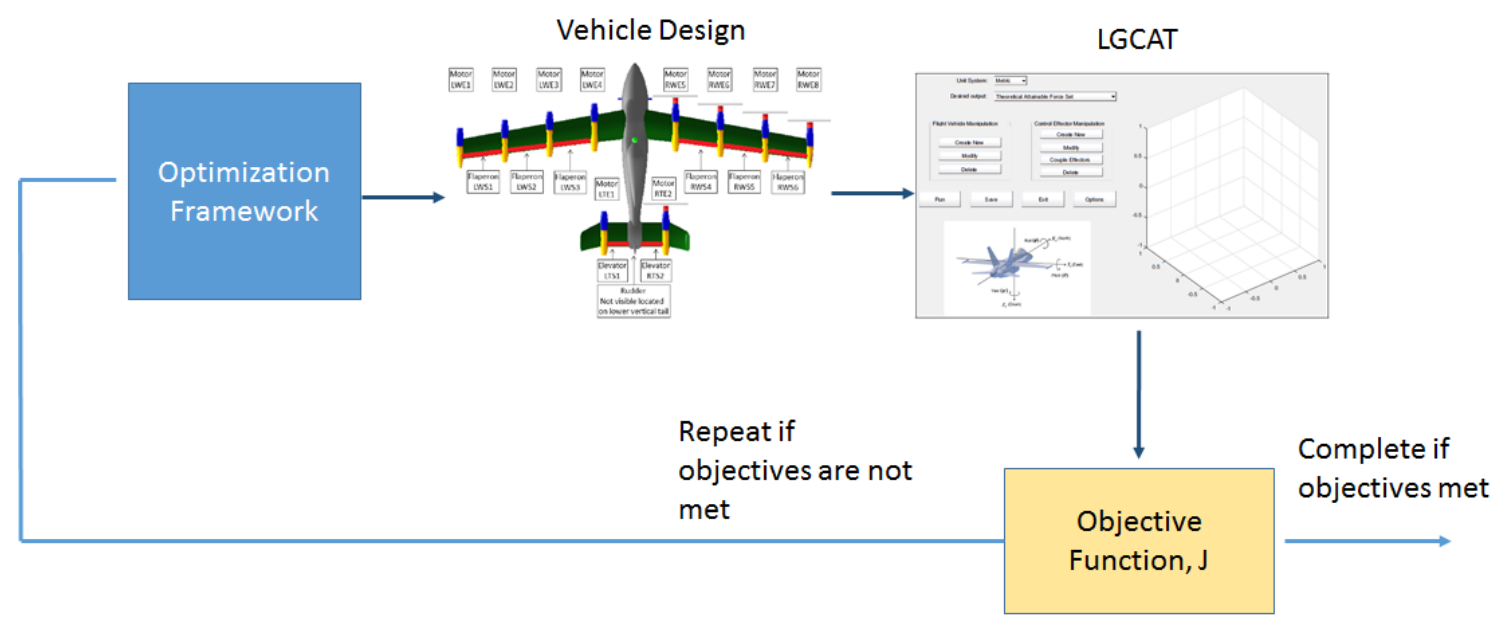

Figure 14: Optimization Framework

The proposed Max Launch Abort System (MLAS) escape vehicle concept ${ }^{15}$ shown in Fig. 15 is an excellent example of how the LGCAT optimization framework can be beneficial to vehicle design. The main objective of the MLAS is to get the crew capsule away from a failed launch vehicle as fast as possible during time of maximum dynamic pressure. The proposed design has six solid rocket escape motors positioned circumferentially at $60^{\circ}$ intervals. Each motor is canted outward $30^{\circ}$. Each escape motor is equipped with thrust vector control and nozzles can be deflected up to $15^{\circ}$ in any direction outward of the fairing (nozzles not allow to deflect inward). To maximize axial acceleration one would minimize the cant angles as much as possible. To maximize the control authority in the pitch and yaw axes one can either increase the cant angle or the gimbal angle capabilities. However, it is not intuitive what the optimum cant angles should be if the design needs to achieve a certain axial acceleration profile and still be robust to uncertainties in the aerodynamic disturbance torques. The LGCAT-based optimization framework described can be used to answer such a design question. Furthermore in the event the design does not meet performance requirements because of constraints on the cant angles, LGCAT can be used to answer questions such as additional gimbal angle range required (beyond the $15^{\circ}$ limit) in order for the system to meet the specified requirements. In this example the optimal solution may appear to be trivial; however, for more complicated configurations, the optimization framework may be necessary to yield an "optimal" design.

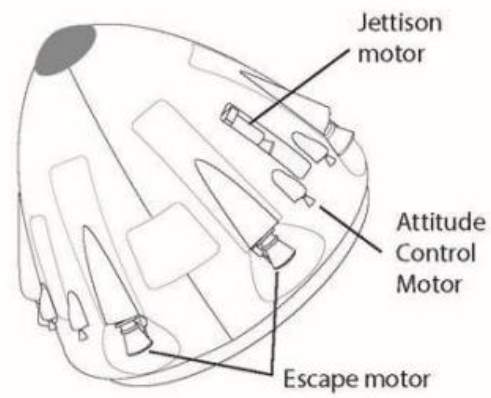

Figure 15: MLAS Escape Vehicle Concept ${ }^{15}$ 


\section{Conclusion}

The development and application of a Generic Control Allocation Toolbox developed at NASA Langley Research Center (LGCAT) is described in this paper. The objective of LGCAT is to provide system engineers and designers early in the vehicle design phase with quick insights on how control effector parameters such as quantity, sizing, location, orientation, redundancy, etc., affect the overall controllability and other performance metrics. Having such information in hand, allows engineers to make more informed decisions on overall mission objectives such as performance vs. reliability vs. cost, etc. early in a vehicle design phase and reduce the number of iterations necessary in the design and analysis cycles. LGCAT can accept a variety of control effector types including aerodynamic surfaces, propellers, thrust vector control engines, and reaction control systems. LGCAT is MATLAB based, user friendly, and is capable of performing the analysis in the Graphical User Interface or script mode. Current add-on features include interfacing with engineering level codes such as Vehicle Sketch Pad and an optimization framework that allows the designer to optimize effector parameters from a controllability standpoint given certain constraints. These capabilities potentially makes LGCAT an integral tool in the preliminary design phase for any aerospace vehicle.

\section{Acknowledgment}

The authors would like to thank NASA Langley Research Center System Analysis and Concept Directorate (SACD) innovation funds to support this work.

\section{References}

${ }^{1}$ Durham, W., "Constrained Control Allocation: Three-Moment Problem," Journal of Guidance, Control, and Dynamics, Vol. 17, No. 2, Mar-Apr 1994.

${ }^{2}$ Orr, J. and Slegers, N., "High Efficiency Thrust Vector Control Allocation," Journal of Guidance, Control, and Dynamics, Vol. 37, No. 2, Mar-Apr 2014.

${ }^{3}$ Durham, W., Bordignon, K., and Beck, R., Aircraft Control Allocation, Wiley, 1st ed., 2017.

${ }^{4}$ Frost, S., "Application of Structural Load Feedback in Flight Control," No. AIAA-2011-6288, AIAA, 2011.

${ }^{5}$ Bodson, M., "Evaluation of Optimization Methods for Control Allocation," No. AIAA-2001-4223, AIAA, 2001.

${ }^{6}$ Glaze, M. and Durham, W., "Design and Implementation of a Control Allocation Toolbox for MATLAB v.5.0," No. AIAA-98-4436, AIAA, 1998.

${ }^{7}$ Linkping University, QCAT - Control Allocation Toolbox, May 2003.

${ }^{8}$ Pei, J., Choueri, M., Elandt, R., Peck, M., , and Finch, P., "An Innovative Control Allocation Scheme to Address Reaction Thruster Interactions on a 3U CubeSat," No. AIAA 2018-2096, AIAA, 2018.

${ }^{9}$ National Aeronautics and Space Administration, Vehicle Sketch Pad, 3rd ed., September 2017.

${ }^{10}$ Massachusetts Institute of Technology, Athena Vortex Lattice Method, November 2007.

${ }^{11}$ Rosema, C. and Doyle, J., Missile DATCOM, U.S. Army Aviation and Missile Research, Development, and Engineering Center, December.

${ }^{12}$ Rothhaar, P., Murphy, P., Bacon, B., Gregory, I., Grauer, J., Busan, R., and Croom, M., "NASA Langley Distributed Propulsion VTOL Tilt-Wing Aircraft Testing, Modeling, Simutlion, Control, and Flight Test Development," No. AIAA-20142999, AIAA, 2014.

${ }^{13}$ Nakano, M. and Willms, R., "Space Shuttle On-Orbit Flight Control System," No. AIAA-1982-38970, AIAA, 1982.

${ }^{14}$ MATLAB, MATLAB Optimization Toolbox, 2017th ed.

${ }^{15}$ Tartabini, P., Gilbert, M., and Starr, B., "Proposed Ascent Abort Flight Test for the Max Launch Abort System," No. AIAA 2016-0783, AIAA, 2016. 